by the product of the diagonal elements, i. e.

$$
|\boldsymbol{C}|=\prod_{\beta_{2} \ldots \beta_{n}} 2^{\beta_{2}} \ldots n^{\beta_{n}} \neq 0 \text {. }
$$

Consequently, the matrix is non-singular and may be inverted to yield all classes of $S_{n}$ as linear combinations of products of the purely cyclic classes.

3. To obtain the projection operators (4) in terms of the SOC's of the cyclic classes $(2),(3), \ldots,(n)$, one has, in principle, to invert the matrix $\boldsymbol{C}$ considered in section 2 . We may, however, make use of the fact that the eigenvalues $\lambda^{u}(i), i=2,3, \ldots, n$ of the SOC's of the cyclic classes suffice to completely characterize bases for the irreducible representation $\mu$ of $S_{n}$. These eigenvalues are given in terms of the characters of $S_{n}$, and we may therefore employ the Löwdin ${ }^{3}$ projection procedure to write

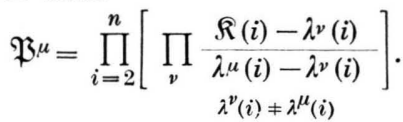

3 P.-O. Löwdin, Rev. Mod. Phys. 36, 966 [1964].
The first product gives the desired factorization, and the full expression obviously yields one for bases of the irreducible representation $\mu$ of $S_{n}$ and zero otherwise. Another way of obtaining the bases would be to diagonalize the $n-1$ operators $\Omega(i), i=2,3, \ldots, n$. Besides the characters of the cyclic classes of $S_{n}$, (10) employs only the matrix elements of the cyclic permutations and therefore reduces considerably the labour involved in the projection procedure. To obtain Yamanouchi bases which transform irreducibly under all groups in the chain $S_{n} \supset S_{n-1} \supset \ldots \supset S_{2}$, the projection may be repeated for each subgroup in the chain.

An important application of this projection technique arises from the consideration of states given in terms of relative coordinates. States of this type result in nuclear physics from the elimination of center of mass motion and from the examination of clustering in the harmonic oscillator shell model ${ }^{4}$.

4 P. Kramer and M. Moshinskx, Nucl. Phys., to appear.

\section{Zum Leitfähigkeitsmechanismus des Selens}

\section{H. EgGERT}

Siemens-Schuckertwerke AG, Berlin-Siemensstadt, Schaltwerk (Z. Naturforschg. 21 a, 658-660 [1966] ; eingegangen am 19. März 1966)

Das Selen wird wegen der exponentiellen Abhängigkeit seiner elektrischen Leitfähigkeit von der Temperatur zu den Halbleitern gerechnet und gehört damit zu den ältesten untersuchten Stoffen der Halbleiterphysik. Trotzdem ist bemerkenswert, daß bis zum heutigen Tage ein umfassendes Modell der elektrischen Leitfähigkeit fehlt. Dies liegt nur zum Teil daran, daß die elektrische Leitfähigkeit eine komplizierte Funktion von Verunreinigungen, Dotierungen, Kristallisationszustand, Temperaturvorgeschichte usw. ist (viele der erheblich differierenden Versuchsergebnisse auf dem Selengebiet haben hier allerdings ihre Ursache). Der Hauptgrund für das bisherige Versagen bei der physikalischen Deutung liegt sicher jedoch daran, daß das Leitfähigkeitsmodell der „freien Stromträger“ (Elektronengasmodell), das die Grundlage der konventionellen Theorien der Halbleiter und Metalle ist, den Verhältnissen beim Selen nicht adäquat ist. So stellen Joffe und REGEL ${ }^{1}$ das Selen in eine Reihe mit einer großen Gruppe von „Halbleitern“, bei denen die elektrischen Eigenschaften nur durch die Nahordnung der atomaren Bausteine zu erklären sind. Dies steht im schroffen Gegensatz zu dem Fernordnungsgedanken der Elektronengastheorie.

1 A. F. Joffe u. A. R. Regel, Progress in Semiconductors, Vol. 4, Heywood \& Company Ltd., London 1960, S. 237.

2 Im Falle der Existenz von Potentialbarrieren um sonst hochleitende Bereiche werden diese Abschätzungen der Beweglichkeit natürlich ungültig.
Diese Stoffe zeigen meist eine exponentiell mit der Temperatur zunehmende Beweglichkeit. Es ist bemerkenswert, daß das Trägergasmodell keinen Streumechanismus mit dieser Temperaturabhängigkeit kennt. Die Verfasser weisen u. a. darauf hin, daß das Modell der freien Stromträger bei allen Stoffen versagen sollte, deren Beweglichkeit kleiner als $100 \mathrm{~cm}^{2} / \mathrm{Vs}$ ist, weil die mittlere freie Weglänge dann kleiner wird als die Wellenlänge eines Elektrons mit thermischer Geschwindigkeit bei Zimmertemperatur. Bei Beweglichkeiten kleiner als $5 \mathrm{~cm}^{2} / \mathrm{Vs}$ wird die mittlere freie Weglänge sogar kleiner als eine Gitterkonstante. Mit einer Beweglichkeit zwischen $10^{-3} \ldots 1 \mathrm{~cm}^{2} / \mathrm{Vs}$ gehört Selen klar in diese Gruppe ${ }^{2}$.

Außer diesen theoretischen Einwänden gegen das Elektronengasmodel gibt es jedoch eine Reihe eindeutiger experimenteller Ergebnisse, die sich durch freie Träger nicht erklären lassen. Dazu gehören besonders die Absorptionsmessungen von Gobrecht und TAusend ${ }^{3}$ im nahen Infrarot. Die Autoren finden im Ausläufer der Grundgitterabsorption zwischen 4 und $23 \mu$ m eine praktisch konstante Absorption, wenn man von einigen charakteristischen scharfen Banden absieht. Dies gilt sowohl für amorphes als auch einkristallines Selen. Wenn freie Defektelektronen im Selen vorhanden wären, dann müßte im untersuchten Einstrahlungsbereich die Absorption der freien Träger mit dem Quadrat der Wellenlänge zunehmen ${ }^{4}$. Das völlige Fehlen dieser Absorption ist ein starker Hinweis auf eine gewisse Bindung der Defektelektronen.

3 H. Gobrecht u. A. Tausend, Z. Phys. 161, 205 [1961]. A. TAusend, Dissertation; Technische Universität Berlin, 1961.

4 T. S. Moss, Optical Properties of Semiconductors, Butterworth, London 1961. 
Zur Deutung eigener Ergebnisse an Seleneinkristallen benutzt PLessner ${ }^{5}$ das Modell der Potentialbarrieren. Danach bewegen sich die Löcher als freie Träger, d.h. ohne Widerstand, längs der periodisch angeordneten Selenkettenmoleküle ${ }^{6}$, müssen dann aber Potentialbarrieren der Größenordnung $0,1 \mathrm{eV}$ überwinden. Über die Natur und den Ort dieser Barrieren macht Plessner keine Angaben, und er kommt auch sonst bei seinen Abschätzungen in gewisse Schwierigkeiten. Trotzdem lassen sich mit diesem Modell zahlreiche Eigentümlichkeiten wie die exponentielle Beweglichkeit und die feldstärkeabhängige Leitfähigkeit des Selens deuten. Die freie Bewegung längs der Selenketten steht jedoch im Widerspruch zu den obigen Absorptionsmessungen. Daher wird das Potentialbarrierenmodell in der PLessnerschen Form heute abgelehnt ${ }^{3}$.

Eine weitere, sehr entscheidend gegen freie Träger sprechende experimentelle Arbeit stammt von JaumanN und Neckenbürger ${ }^{8}$. Bei DK-Messungen im Frequenzbereich von $30 \ldots 4300 \mathrm{MHz}$ ergibt sich an polykristallinen Proben eine Zunahme der Wechselstromleitfähigkeit mit $v^{1 / 2} \quad(v=$ Frequenz), ohne daß sich eine Sättigung andeutet! Da auch die lange getemperten Proben, deren Kristallitgröße erfahrungsgemäß im Mikrometerbereich liegt, dieses Verhalten zeigen, kommt man auch hier mit dem obigen Potentialbarrierenmodell in Schwierigkeiten. Beim Vorhandensein freier Träger innerhalb der Selenketten müßte sich die Leitfähigkeit bei erheblich kleineren Frequenzen sättigen.

Aus sehr sauberen Wechselspannungsmessungen $\left(10^{3} \ldots 10^{6} \mathrm{~Hz}\right)$ an polykristallinem Selen bestimmen Ludwig und EcKart ${ }^{9}$ die komplexe DK als Funktion der Bromdotierung, Temperzeit und Probentemperatur. Die Analyse der Meßergebnisse führt die Autoren zu dem Schluß, daß nur die Fröhlichsche Theorie der Platzwechselvorgänge ${ }^{10}$ eine vollständige Beschreibung gestattet. Die Platzwechseltheorie geht von der Voraussetzung aus, daß die die Dispersion verursachenden Ladungen oder Dipole mehrere Gleichgewichtslagen besitzen, die durch Potentialbarrieren voneinander getrennt sind. Als Ort der Platzwechselvorgänge nimmt LuDwig ${ }^{9}$ Übergänge an den Kettenenden an. Mit diesen Annahmen läßt sich besonders gut der Befund deuten, daß sowohl die elektrische Leitfähigkeit als auch die Frequenz im Maximum der dielektrischen Verluste (reziproke Relaxationszeit) einem Exponentialgesetz der Temperatur mit der gleichen Aktivierungsenergie folgen. Beiden Erscheinungen liegt also der gleiche physikalische Mechanismus zugrunde. Über die Ver-

5 K. W. Plessner, Proc. Phys. Soc. London B 64, 671 [1951].

6 Das Selen kristallisiert in schraubenförmigen Kettenmolekülen, deren Achsen sich hexagonal anordnen (vgl. z. B. v. HIPPEL ${ }^{7}$ ).

7 A. v. Hippel, J. Chem. Phys. 16, 372 [1948].

8 J. Jaumann u. E. Neckenbürger, Z. Phys. 151, 72 [1958].

9 W. Ludwig, Dissertation, Humboldt-Universität Berlin 1960. - W. Ludwig u. F. Eckart, Z. Phys. 162, 127 [1961].

$10 \mathrm{H}$. F RöHLich, Theory of Dielectrics, At the Clarendon Press, 2. Aufl., Oxford 1958.

11 M. Pollak u. T. H. Geballe, Phys. Rev. 122, 1742 [1961]. hältnisse längs der Selenketten wird keine Angabe gemacht.

Die Ergebnisse beider Autorengruppen gestatten die folgende Interpretation. LUDwig und ECKART weisen das Potentialbarrierenmodell mit ihren Dispersionsmessungen bei niedrigen Frequenzen direkt nach und bestätigen seine Gültigkeit auch für die Leitfähigkeit. JAumanN und Neckenbürger finden bei sehr hohen Frequenzen, daß die Bewegung der Defektelektroden längs der Selenketten nicht frei sein kann. Die ständige Zunahme der Wechselstromleitfähigkeit mit der Frequenz läßt sich nur so deuten, daß die Bewegung der Defektelektronen längs der Ketten ein Hüpfprozeß ist (engl. Hopping) ${ }^{11}$. Damit meint man, daß sich das Defektelektron mittels eines thermisch aktivierten Sprungprozesses von einem Gitterbaustein zum anderen bewegt, da $\beta$ es sonst aber in einer Potentialmulde gebunden ist. Diese Bewegung wird bevorzugt längs der Kette erfolgen. Sprünge von einer Kette zur benachbarten müssen beim Selen ebenfalls eine nicht zu vernachlässigende Wahrscheinlichkeit haben, weil die Querleitfähigkeit senkrecht zur $c$-Achse nur um den Faktor drei bis vier kleiner ist als die Leitfähigkeit in Richtung der $c$-Achse ${ }^{12}$. Für die Deutung durch den Hüpfprozeß spricht auch die bei JaUmann und Neckenbürger offensichtliche Konvergenz der DK-Werte bei hohen Frequenzen. Damit deutet sich eine vom Kristallisationszustand des Selens unabhängige Eigenschaft an, deren Ursache nur die stets vorhandene Nahordnung der Selenatome innerhalb der Kette sein kann.

Es liegt nun der Schluß sehr nahe, beide Ergebnisse zu kombinieren. Dies erscheint schon deshalb unproblematisch, weil erstens die Frequenzbereiche relativ weit

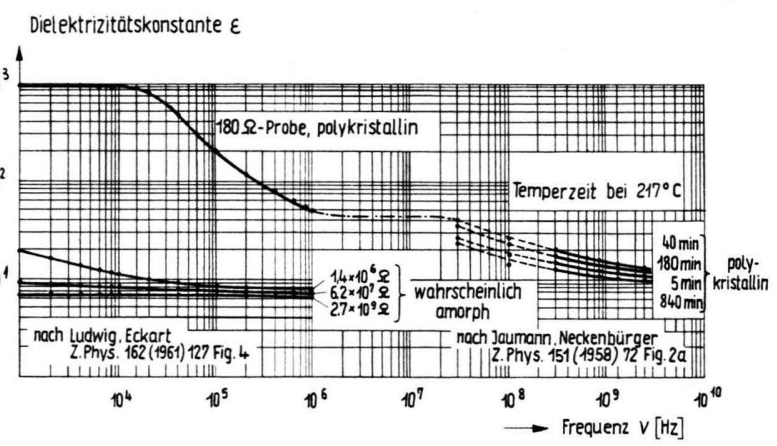

Abb. 1. Dielektrizitätskonstante des Selens als Funktion der Frequenz. Kombination der Meßergebnisse von Ludwig, Eckart und Jaumane, Neckenbürger (vgl. ${ }^{13}$ ).

12 H. W. Henkels, J. Appl. Phys. 22, 916 [1951]. - H. W. Henkels u. J. Maczuk, Phys. Rev. 91, 1562 [1953].

13 Bei dem niederfrequenten Teil handelt es sich um die einzigen Kurven aus der Arbeit von Ludwig und Eckart, die bei Zimmertemperatur gemessen wurden. Über den $\mathrm{Zu}$ stand des Selens finden sich keine Angaben.

Die Kurven von Jaumann und Neckenbürger sind mit Reinstselen gewonnen. Die gestrichelten Kurventeile sind ebenfalls übernommen - sie sind nach den Verfassern wegen einer anderen Meßmethode mit größeren Unsicherheiten behaftet. 
auseinander liegen und damit unterschiedliche Mechanismen erfaßt werden und weil sich zweitens ein gewisser Anschluß der Meßergebnisse als möglich erweist. Das Zentrum der Relaxationszeit liegt bei LuDwig und Eckart zwischen $10^{-5}$ und $10^{-6}$ sec und bei JaUmanN und Neckenbürger bei $10^{-9} \mathrm{sec}$, wobei der letztere Wert nach Angabe der Autoren als sehr unsicher gelten muß. Vergleicht man die DK-Werte (unter Berücksichtigung eines Wertes $C_{0}=7 \mathrm{pF}^{9}$ ), dann passen sie sich im nicht ausgemessenen $Z$ wischenbereich gut einander an (Abb. 1) ${ }^{13}$. Beide Ergebnisse lassen sich also als doppelte DK-Stufe interpretieren. Die erste DK-Stufe bei kleinen Frequenzen entsteht durch die Platzwechselvorgänge über die Potentialbarrieren an

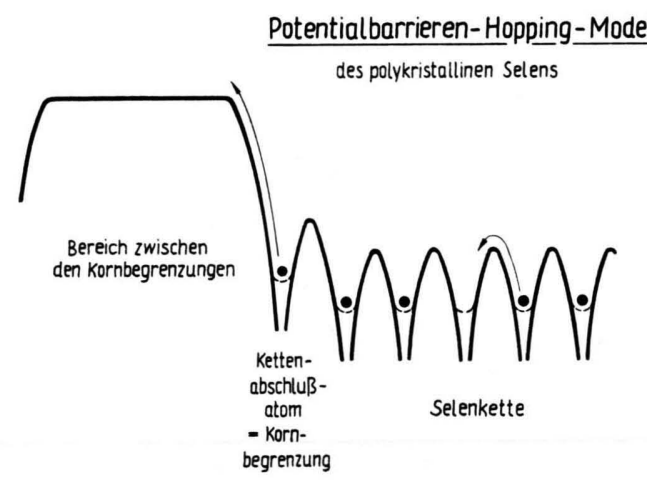

Abb. 2. Schematische Darstellung des Potentialverlaufes innerhalb der Selenkette und am Kettenende.

14 J. Yamashita u. T. Kurosawa, J. Phys. Soc. Japan 15, 802 [1960].

15 A. Miller u. E. Abrahams, Phys. Rev. 120, 745 [1960].

16 G. L. Sewell, Phys. Rev. 129, 597 [1963].

17 T. Holstein, Ann. Phys. (N. Y.) 8, 325, 343 [1959]. L. Friedman u. T. Holstein, Ann. Phys. (N. Y.) 21, 494 [1963]. den Kettenenden, wobei die Defektelektronen genügend Zeit haben, die Kette zu durchlaufen. Die zweite DKStufe bei hohen Frequenzen entsteht durch den Hüpfprozeß über die Kettenglieder.

Der dominierende Charakter der Potentialbarrieren an den Kettenenden für die elektrische Leitfähigkeit und die Dispersion bei niedrigen Frequenzen läßt sich nur dann verstehen, wenn man annimmt, daß sie energetisch höher sind als die Aktivierungsenergie des Hüpfprozesses. Das daraus folgende Potentialschema unseres Leitfähigkeitsmodells zeigt Abb. 2.

Das vorgeschlagene Modell gestattet es, zahlreiche Untersuchungen und Meßergebnise am Selen von einem einheitlichen Standpunkt aus zu deuten. Die völlige Abkehr von dem konventionellen Halbleiterbild wird gerechtfertigt durch die bisherigen vergeblichen Versuche, das Selen auf dieser Basis zu deuten. Das Hüpfmodell ist in den letzten Jahren als wichtige Alternative zum Trägergasmodell zunehmend das Ziel theoretischer Untersuchungen ${ }^{14-17}$ und kann daher unbedenklich als gültiges Modell angesehen werden. $\mathrm{Da} ß$ zahlreiche Stoffe diesen Leitungsmechanismus zeigen, kann ebenfalls nicht mehr bezweifelt werden ${ }^{1,11,18,19}$.

Abschließend bleibt zu bemerken, daß das Potentialbarrieren-Hopping-Modell nicht nur aus der Analyse der HF-Messungen folgt, sondern auch aus den Ergebnissen eigener Dotierungsuntersuchungen an polykristallinem Selen. Eine ausführliche Darstellung dieser Arbeiten sowie aller mit dem Leitungsmodell zusammenhängenden Fragen liegt vor ${ }^{20}$. Weitere Veröffentlichungen sind vorgesehen.

18 G. H. Jonker u. S. v. Houten, Halbleiterprobleme Band VI, Verlag Vieweg, Braunschweig 1961, S. 118.

19 F. J. Morin, Bell System Techn. J. 37, 1047 [1958] bzw. N. B. Hannay, Semiconductors, Reinhold Publishing Corp., New York 1959, S. 600.

20 H. EgGERT, Dissertation, Technische Universität Berlin 1965. 